\author{
Marquette University \\ e-Publications@Marquette
}

Electrical and Computer Engineering Faculty

Research and Publications

Electrical and Computer Engineering,

Department of

2001

\title{
Impact-ionization and noise characteristics of thin III-V avalanche photodiodes
}

\author{
Mohammad A. Saleh \\ University of Dayton \\ Majeed M. Hayat \\ Marquette University, majeed.hayat@marquette.edu \\ Paul P. Sotirelis \\ University of Illinois - Urbana-Champaign \\ Archie L. Holmes \\ University of Texas - Austin \\ Joe C. Campbell \\ University of Texas - Austin
}

See next page for additional authors

Follow this and additional works at: https://epublications.marquette.edu/electric_fac

Part of the Computer Engineering Commons, and the Electrical and Computer Engineering Commons

\section{Recommended Citation}

Saleh, Mohammad A.; Hayat, Majeed M.; Sotirelis, Paul P.; Holmes, Archie L.; Campbell, Joe C.; Saleh, Bahaa E.A.; and Teich, Malvin Carl, "Impact-ionization and noise characteristics of thin III-V avalanche photodiodes" (2001). Electrical and Computer Engineering Faculty Research and Publications. 520.

https://epublications.marquette.edu/electric_fac/520 


\section{Authors}

Mohammad A. Saleh, Majeed M. Hayat, Paul P. Sotirelis, Archie L. Holmes, Joe C. Campbell, Bahaa E.A. Saleh, and Malvin Carl Teich 
Marquette University

e-Publications@Marquette

\section{Electronic and Computer Engineering Faculty Research and Publications/College of Engineering}

This paper is NOT THE PUBLISHED VERSION; but the author's final, peer-reviewed manuscript. The published version may be accessed by following the link in the citation below.

IEEE Transaction on Electron Devices, Vol. 48, No. 12 (2001): 2722-2731. DOI. This article is (C) Institute of Electrical and Electronic Engineers (IEEE) and permission has been granted for this version to appear in e-Publications@Marquette. Institute of Electrical and Electronic Engineers (IEEE) does not grant permission for this article to be further copied/distributed or hosted elsewhere without the express permission from Institute of Electronic and Electrical Engineers (IEEE).

\section{Impact-ionization and noise characteristics of thin III-V avalanche photodiodes}

Mohammad A. Saleh

University of Dayton

Majeed M. Hayat

University of New Mexico

Paul P. Sotirelis

University of Illinois at Urbana-Champaign

Archie L. Holmes

University of Texas at Austin 
Joe C. Campbell

\title{
University of Texas at Austin
}

\author{
Bahaa E. A. Saleh \\ Boston University \\ Malvin Carl Teich
}

Boston University

\section{Abstract:}

It is, by now, well known that McIntyre's localized carrier-multiplication theory cannot explain the suppression of excess noise factor observed in avalanche photodiodes (APDs) that make use of thin multiplication regions. We demonstrate that a carrier multiplication model that incorporates the effects of dead space, as developed earlier by Hayat et al. provides excellent agreement with the impact-ionization and noise characteristics of thin $\mathrm{InP}, \mathrm{In} / \mathrm{sub} 0.52 / \mathrm{Al} / \mathrm{sub} 0.48 / \mathrm{As}$, GaAs, and Al/sub 0.2/Ga/sub 0.8/As APDs, with multiplication regions of different widths. We outline a general technique that facilitates the calculation of ionization coefficients for carriers that have traveled a distance exceeding the dead space (enabled carriers), directly from experimental excess-noise-factor data. These coefficients depend on the electric field in exponential fashion and are independent of multiplication width, as expected on physical grounds. The procedure for obtaining the ionization coefficients is used in conjunction with the dead-space-multiplication theory (DSMT) to predict excess noise factor versus mean-gain curves that are in excellent accord with experimental data for thin III-V APDs, for all multiplication-region widths.

\section{SECTION I.}

\section{Introduction}

Recent advances in the design and fabrication of avalanche photodiodes (APDs) have allowed these devices to achieve levels of gain-bandwidth product and array configurability that make them excellent choices for use in current $10 \mathrm{~Gb} / \mathrm{s}$ receivers operating in the silica-fiber window. Within an operational frequency range corresponding to that of current lightwave systems, APDs provide an advantage over p-i-n detectors because of the internal gain that they provide [1]. This gain is, however, accompanied by excess noise that arises from randomness in the coupled avalanching process of the very electrons and holes that give rise to the gain in the first place [2]. It has recently been demonstrated that the use of thin (less than $1 \mu \mathrm{m}$ ) APD multiplication regions serves to reduce excess noise [3], [4]. When operated in appropriate configurations, such as resonant-cavity enhanced (RCE) devices, these APDs can achieve high quantum efficiency ( $>0.7)$ and large gain-bandwidth product (> 290 GHz) [5], [6].

APD noise is most readily characterized by a quantity called the excess noise factor $F$ [1], [2]. Mclntyre [7] first obtained a mathematical form for this function in a classic paper published in 1966. He showed that the excess noise factor depends only on the mean gain $\langle G\rangle$ and on the ratio $k$ of the ionization coefficients for holes and electrons. McIntyre's formula rests on two assumptions: 1 ) that the avalanche multiplication region is uniform and 2) that the ability of electrons and holes to effect an impact-ionization does not depend on their past 
history. The Mclntyre formula has been successfully used for many years to characterize the multiplication noise of conventional thick APDs.

If either (or both) of the above conditions are not satisfied, however, a more general theory is required for calculating the excess noise factor. Nonuniform multiplication regions can be constructed using arbitrary multilayer APD structures created with the help of bandgap engineering. Multiquantum-well APDs [8], [9] are designed such that the carrier multiplication process takes place only at certain preferred locations in the material, as determined by the externally engineered superlattice. The uncertainty in the carrier birth locations is thereby reduced, and a theory admitting nonuniform multiplication [10] is required to describe the noise of these devices. The expression for the excess noise factor will then depend not only on $\langle G\rangle$ and on $k$, but also on the detailed structure of the device [10], [11].

The effect of past history on the ability of a carrier to create a new carrier pair via impact-ionization can also be taken into account, as has been shown previously by Okuto and Crowell [12], La Violette and Stapelbroek [13], Hayat et al. [14] [15]-[16] [17], Spinelli and Lacaita [18], and McIntyre [19]. For APDs with thin multiplication regions [3], [4], [20]-[21][22][23] this turns out to be necessary. Incorporating carrier history is important because newly born carriers are incapable of immediately causing impact-ionizations. They must first travel a sufficient distance (called the dead space), in the course of which they gain enough energy from the field to permit them to cause an impact-ionization. These carriers are then said to be enabled [4].

Gain fluctuations, and therefore APD noise, are typically lowered when the multiplication region is tailored to a nonuniform profile, and when carrier history is taken into account, since both of these features generally reduce randomness. Although neither is accommodated by the McIntyre theory [7], [24], it is often used anyway, with the result that the device characteristics are not properly understood. In the early 1990s, Hayat et al. [14][15][16] formulated a dead-space-multiplication theory (DSMT) that permitted the gain, excess noise factor, and gain probability distribution to be determined in the presence of dead space. Because the DSMT developed in [16] incorporates a nonuniform electric field, it can accommodate arbitrary history-dependent ionization coefficients. The results presented in [15] and [16] clearly illustrated that dead space reduces the excess noise factor, and that this reduction becomes more significant as the ratio of the dead space to the multiplicationregion width increases.

In subsequent years, experiments and Monte Carlo $(\mathrm{MC})$ simulations revealed that the excess noise factor does indeed decrease as the multiplication-region width is reduced [25]-[26][27][29]. Moreover, it has recently been shown [4], [20], [21] that in thin APDs, the dead space represents a significant fraction of the multiplicationregion width (up to $25 \%$ for devices with widths $<100 \sim \mathrm{nm}$ ) and that dead space does indeed play an important role in reducing the excess noise factor. It is principally for this reason that there has been a great deal of interest of late in thin-multiplication-region APDs.

Even with the success of the DSMT in mathematically characterizing the effect of dead space on avalanche multiplication, its utility in predicting experimental results remained limited, primarily due to the absence of a suitable way of determining the ionization coefficients in the presence of dead space. In particular, ionization coefficients extracted from the Mclntyre multiplication theory are not applicable to devices in which dead space plays a role. The DSMT requires knowledge of the ionization coefficients of enabled carriers, i.e., those that have traveled a distance exceeding the dead space. In the absence of a proper theory for the ionization coefficients, Li et al. [20] used a mean-free-path formulation to estimate how the effective ionization coefficients extracted with the help of the McIntyre theory (such as those reported in [30] and [31]) could be modified; they then proceeded to use these modified values in the DSMT to predict the excess-noise characteristics of thin APDs. This approach is, however, approximate, as pointed out by Spinelli et al. [32], and as recognized by Li et al. [20]. 
McIntyre [19] and Yuan et al. [3], [22] subsequently developed a modification of the DSMT and utilized it to study the excess noise factor for thin APDs. The formulation developed in [19] uses a so-called historydependent electric field to incorporate nonlocal effects in the ionization coefficients. However, the implementation of this approach, carried out in [3] and [22], makes use of a free parameter (the width of the Gaussian correlation function used in the formulation of the history-dependent electric field) to facilitate achieving agreement between theory and experiment.

More recently, Saleh et al. [4] directly applied the DSMT to published experimental data for GaAs and AlGaAs APDs [28] and developed a model for the ionization coefficients that was independent of the multiplicationregion width. The formulation in [4] utilized only knowledge of the electric field, the ionization threshold energies for each carrier, and noise-versus-gain experimental data. In particular, for each carrier and each material, a single electric-field-dependent model for the ionization coefficient was developed that was suitable for devices of all thicknesses ( $100 \mathrm{~nm}-1600 \mathrm{~nm}$ for the particular data sets examined). In essence, the paper by Saleh et al. [4], published in 2000, provided a methodology, as well as a practical procedure, for calculating the material-specific ionization coefficients for carriers that have traveled a distance greater than the dead space, directly from gain and noise measurements. This feature distinguishes their approach [4] from other variations [3], [20], [22] of the original DSMT implementation [15].

When this width-independent model for carrier ionization was used in conjunction with the DSMT to calculate the excess noise factor, good agreement with experimental results were obtained [4]. The fits, however, were found to be sensitive to the selection of the electron and hole ionization threshold energies, which are key parameters in the DSMT. Unfortunately, accurate theoretical estimates of the ionization threshold energies have been reported only for a limited number of materials, specifically for electrons in GaAs and InP [33], and the threshold energies reported elsewhere are of questionable accuracy. By virtue of the free parameter in their approach, however, the predictions obtained by Yuan et al. [3], [22] were superior. A way had to be found to adjust the theoretical ionization threshold energies, resulting in effective threshold energies, to better accommodate the presence of dead space and the underlying physics into the model.

In this paper, we do precisely that. In optimizing the fit of theory to data, the ionization-threshold energies are permitted to deviate from the theoretical values reported in the literature. This optimization process essentially extracts the effective ionization threshold energies. The advantage of adjusting the ionization threshold energy in APD noise prediction was originally highlighted by Li et al. [20] in the context of their approach, which differs in principle from ours [4], as discussed earlier.

The enhanced procedure reported here turns out to provide substantially improved agreement of the DSMT calculations with experiment, while maintaining all of the underlying physical principles attendant to the DSMT model [4], [15]. This is illustrated by comparing the outcome provided by the new procedure outlined here with that used previously [4] for thin-multiplication-region GaAs and AIGaAs APDs. InP and InAIAs APDs with various multiplication-region widths are also examined in detail. The agreement of the DSMT with experiment turns out to be excellent for all four materials, for all multiplication-region widths examined.

\section{SECTION II.}

\section{Dead-Space Multiplication Theory}

For completeness, we begin by reviewing some germane aspects of the double-carrier multiplication DSMT [15]. Dead space is a feature of the avalanche-multiplication process because band-to-band impact-ionization can take place only after an electron or hole has acquired sufficient kinetic energy to collide with the lattice and ionize another electron-hole pair. The smallest value of the ionizing-particle kinetic energy that can accommodate this process is termed the ionization threshold energy, denoted Eie and Eihfor the electron and 
hole, respectively. The minimum distance that a newly generated carrier must travel in order to acquire this threshold energy is termed the carrier dead space [12], and is denoted de and $d \mathrm{~h}$ for electrons and holes, respectively. Assuming the absence of phonon scattering, the presence of a uniform electric field $E$ in the multiplication region gives rise to a constant force so that [12]

(1) Top

(2) Bottom

$$
\begin{aligned}
& d_{\mathrm{e}}=\frac{E_{\mathrm{ie}}}{q \mathcal{E}} \\
& d_{\mathrm{h}}=\frac{E_{\mathrm{ih}}}{q \mathcal{E}}
\end{aligned}
$$

where $q$ is the electronic charge.

The use of these equations in the presence of phonon scattering would likely result in larger estimates of the effective ionization threshold energies than those returned by theoretical estimates, which do not involve phononscattering effects. And modeling phononscattering loss is a difficult enterprise. However, it has been argued by Anderson and Crowell [34], for example, that the relative value of the phononscattering loss is significantly smaller than the ionization threshold energy. Thus it has been customary to assume zero phononscattering loss [16], [20], [21], and we continue in this tradition.

The gain statistics for double-carrier multiplication APDs, in the presence of dead space and a uniform or a nonuniform electric field, have been developed and reported in [15], [16]. The theory involves recurrence equations of certain intermediate random variables $Z(x)$ and $Y(x)$. The quantity $Z(x)(Y(x))$ is defined as the overall electron and hole progeny generated by a single parent electron (hole) at the position $x$ in the multiplication region, which is assumed to extend from $x=0$ toward $x=W$. The electric field within the multiplication region is assumed to be pointing from $x=W$ to $x=0$. In the case of electron injection at the edge of the multiplication region, the random gain $G$ of the APD is simply $(Z(0)+Y(0)) / 2$, which can be further reduced to $G=(Z(0)+1) / 2$ since $Y(0)=1$. According to [15], the averages of $Z(x)$ and $Y(x)$, denoted by $z(x)$ and $y(x)$, respectively, obey the following set of coupled integral equations:

(3) Top

(4) Bottom

$$
\begin{array}{lc}
z(x)= & {\left[1-\int_{0}^{W-x} h_{\mathrm{e}}(\xi) d \xi\right]} \\
& +\int_{x}^{W}[2 z(\xi)+y(\xi)] h_{\mathrm{e}}(\xi-x) d \xi \\
y(x)= & {\left[1-\int_{0}^{x} h_{\mathrm{h}}(\xi) d \xi\right]} \\
& +\int_{0}^{x}[2 y(\xi)+z(\xi)] h_{\mathrm{h}}(x-\xi) d \xi .
\end{array}
$$

Here, $h_{\mathrm{e}}(x)$ and $h_{\mathrm{h}}(x)$ are the probability density functions (pdfs) of the random free-path lengths $X_{\mathrm{e}}$ and $X_{\mathrm{h}}$ of the electron and hole, respectively.

Defining $\alpha$ and 6 , respectively, as the ionization coefficients of enabled electrons and holes, i.e., those that have traveled a distance greater than the dead space, a plausible model for the above pdfs is given by [15] 


$$
\begin{aligned}
& h_{\mathrm{e}}(x)=\alpha e^{-\alpha\left(x-d_{\mathrm{e}}\right)} u\left(x-d_{\mathrm{e}}\right) \\
& h_{\mathrm{h}}(x)=\beta e^{-\beta\left(x-d_{\mathrm{h}}\right)} u\left(x-d_{\mathrm{h}}\right)
\end{aligned}
$$

where $u(x)$ is the unit step function [ $u(x)=1$ if $x \geq 0$, and $u(x)=0$ otherwise]. These pdfs correspond to a fixed (hard-threshold) dead space, for which the ionization coefficient of a carrier is zero for carriers that have traveled a distance shorter than the dead space and is constant for carriers that have traveled a longer distance.

This model is the spatial analog of the fixed nonparalyzable dead-time-modified Poisson process [35]-[36][37]; the underlying Poisson character of this process is responsible for the exponential behavior of the inter-event intervals in (5) and (6) for distances greater than the dead space. In some physical processes the dead space is stochastic, rather than fixed, which gives rise to the variable nonparalyzable dead-time-modified Poisson process [38]. In other circumstances, carriers can be gradually, rather than abruptly, enabled, resulting in relative (soft-threshold) dead space, which is the spatial analog of the sick-time-modified Poisson process [39]. MC simulation shows that APD carriers do indeed exhibit relative dead space (sick space); the ionization probability is low following birth and gradually increases over some distance [23]. Although sick space provides a superior representation of physical reality than does dead space, the fixed dead-space model adequately captures the essential history dependence of the impact-ionization process and does so without the necessity of postulating an adhoc recovery function. And, it has the salutary feature of being amenable to far simpler analysis.

Using (5) and (6) to obtain solutions to $\underline{(3)}$ and $\underline{(4)}$, then, leads to a mean gain

$$
\langle G\rangle=\frac{z(0)+1}{2}
$$

and an excess noise factor given by

$$
F \equiv \frac{\left\langle G^{2}\right\rangle}{\langle G\rangle^{2}}=\frac{z_{2}(0)+2 z(0)+1}{[z(0)+1]^{2}} .
$$

Here, $z_{2}(x)=\left\langle Z^{2}(x)\right\rangle$ and $y_{2}(x)=\left\langle Y^{2}(x)\right\rangle$ are the second moments of $Z(x)$ and $Y(x)$, respectively. According to [15], these quantities are, in turn, governed by the following set of coupled integral equations: 


$$
\begin{gathered}
\quad\left[1-\int_{0}^{W-x} h_{\mathrm{e}}(\xi) d \xi\right] \\
+\int_{x}^{W}\left[2 z_{2}(\xi)+y_{2}(\xi)+4 z(\xi) y(\xi)+2 z^{2}(\xi)\right] \\
\quad \times h_{\mathrm{e}}(\xi-x) d \xi \\
y_{2}(x)=\quad\left[1-\int_{0}^{x} h_{\mathrm{h}}(\xi) d \xi\right] \\
+\int_{0}^{x}\left[2 y_{2}(\xi)+z_{2}(\xi)+4 z(\xi) y(\xi)+2 y^{2}(\xi)\right] \\
\quad \times h_{\mathrm{h}}(x-\xi) d \xi .
\end{gathered}
$$

The recurrence equations (3), (4), (9), and (10) can be solved to estimate $\langle G\rangle$ and $F$ by using a simple iterative numerical recipe (Picard iterations), as outlined in [15]. Given the electric field and ionization threshold energies, one can search for the values of $\alpha$ and $B$ that yield a specified mean gain and excess noise factor.

Hole-injection APDs are analyzed by interchanging the electron and hole ionization coefficients, the electron and hole dead spaces, and the $z s$ and $y s$.

The dependence of the electron and hole ionization coefficients on the electric field $\mathrm{E}, \alpha(\mathcal{E})$ and $\beta(\varepsilon)$, respectively, is often modeled by the standard equation [40], [41]

$$
\alpha(\mathcal{E}), \beta(\varepsilon)=A \exp \left[-\left(\frac{\mathcal{E}_{c}}{\mathcal{E}}\right)^{m}\right]
$$

where $A, \mathcal{E}_{c}$, and $m$ are parameters chosen by fitting measured excess-noise-factor data [30], [42]. We adopt the same equation to model the ionization coefficients of enabled carriers, which are determined as follows: After calculating a unique pair of electron and hole ionization coefficients for a mean-gain and excess-noise-factor experimental pair corresponding to a specific electric field $\mathcal{E}$ we obtain the parameters $A, \mathcal{E}_{c}$, and $m$ for both electrons and holes over a range of electric-field values and multiplication-region widths by fitting the ionization coefficients to the model provided in (11). In Section III, a search algorithm is outlined that exploits this scheme to estimate the ionization coefficients of enabled carriers.

\section{SECTION III.}

\section{Dead-Space Multiplication Theory and the Excess Noise Factor}

To demonstrate the applicability of the DSMT to APDs with thin multiplication regions of various widths, we analyze experimental excess-noise-factor data for InP, InAIAs, GaAs, and AlGaAs separate-absorptionmultiplication (SAM) homojunction devices [3]. The devices consist of four InP APDs with multiplication-region widths of $281 \mathrm{~nm}, 317 \mathrm{~nm}, 582 \mathrm{~nm}$, and $1110 \mathrm{~nm}$; four $\mathrm{In}_{0.52} \mathrm{Al}_{0.48}$ As APDs with widths of $190 \mathrm{~nm}, 363 \mathrm{~nm}, 566$ $\mathrm{nm}$, and $799 \mathrm{~nm}$; four GaAs APDs with widths of $100 \mathrm{~nm}, 200 \mathrm{~nm}, 500 \mathrm{~nm}$, and $800 \mathrm{~nm}$; and three Al0.2Ga0.8As APDs with widths of $200 \mathrm{~nm}, 400 \mathrm{~nm}$, and $800 \mathrm{~nm}$. Portions of these data have been published previously [3], [28]. DSMT analyses of some of these data have been carried out by Yuan et al. [22], using the particular DSMT approach presented in [19], and also by Saleh et al. [4]. 
A. Procedure for Determining the Universal Model for the Ionization Coefficients of Enabled Carriers The width-independent (universal) ionization coefficients are determined as follows:

1. Given knowledge of the electric field and the width of the multiplication region, along with the ionization threshold energies, the electron and hole dead spaces are computed using (1) and (2).

2. For each experimental mean-gain and excess-noise-factor pair, appropriate values for $\alpha$ and $B$ are determined from the recurrence equations that lead to the DSMT mean gain and excess noise factor. This step is carried out by inserting initial values for the ionization coefficients in the recurrence equations and subsequently varying these values until the DSMT mean gain and excess noise agree with the measured values.

3. The previous step is repeated, spanning all measurements of the experimental mean gain and excess noise factor at different values of the electric field and for multiplication regions of different widths.

4. When all excess-noise-factor data points are exploited, each resulting ionization coefficient is used in the standard exponential model [given by (11)] to determine the parameters of this model using a leastsquare-error fit. These four steps are essentially identical to those outlined in [4]. The models for the electron and hole ionization coefficients are used to predict the dependence of the excess noise factor on the mean gain.

However, the fit to excess-noise-factor curves can be substantially enhanced by carrying out an additional step.

5. The first four steps are repeated while adjusting the electron and hole ionization thresholds over a narrow range until the best excess noise factor versus mean-gain prediction is obtained.

This procedure is warranted on physical grounds, as discussed earlier. This procedural modification of implementing the DSMT model is the principal contribution of this paper. Its efficacy will be demonstrated in Section IV. 


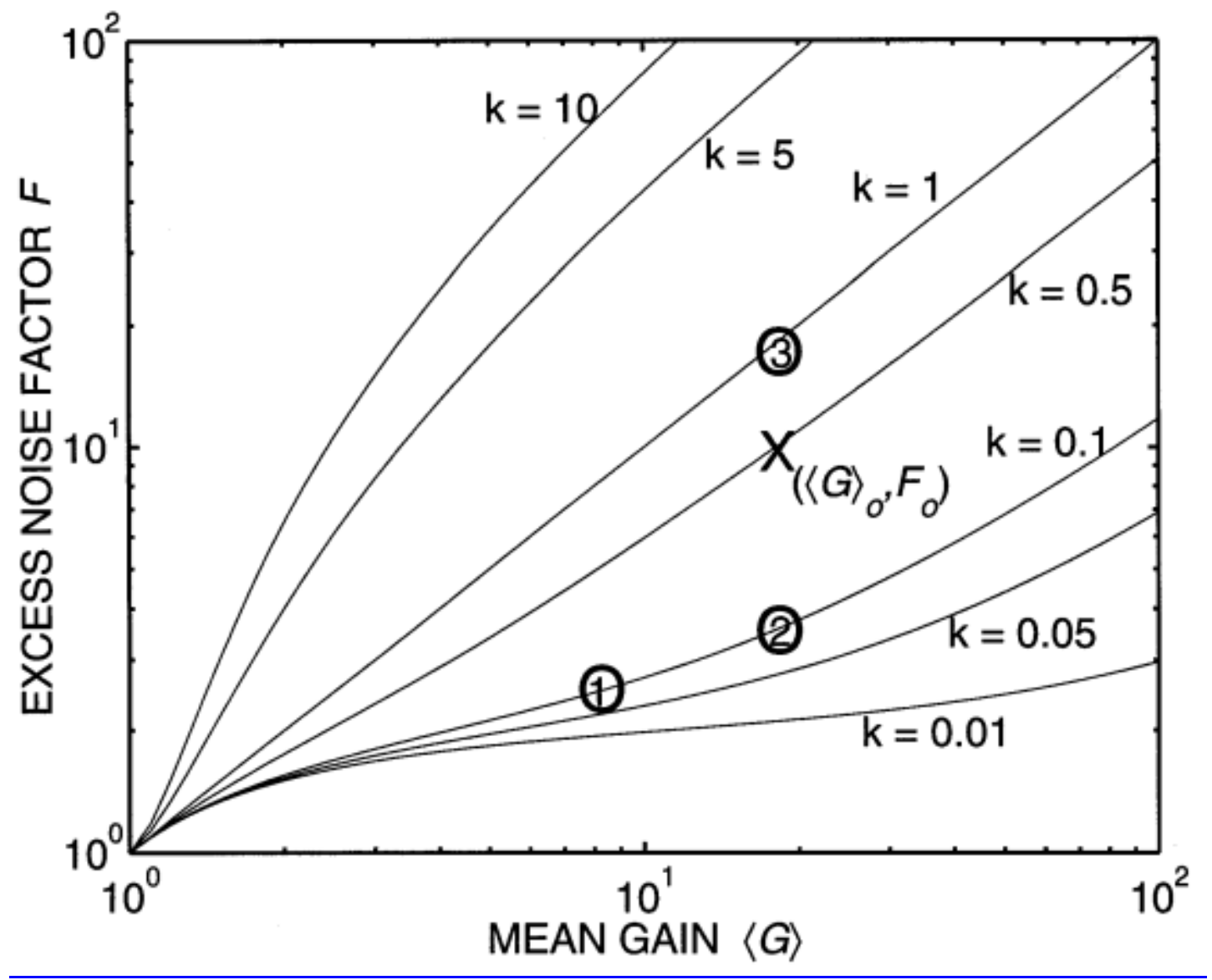

Fig. 1. General characteristics of the excess noise factor $(F)$ versus the mean gain $(\langle G\rangle)$ parameterized by the hole-to-electron ionization ratio $k$, according to the conventional Mclntyre theory [7]. The encircled points 1, 2, and 3 are samples of the $(\langle G\rangle, F)$ values generated in the process of finding the ionization coefficients of enabled carriers using the dead-space theory.

Since Step 2 is critical in implementing the DSMT model, we review the procedures involved in carrying it out for the case of electron-edge injection [4]. To save computational time, we initially use trial values for the electron ionization coefficient and the hole-to-electron ionization ratio determined from the conventional McIntyre theory which has the merit of simple closed-form expressions. These initial values are denoted $\alpha=\alpha 0$ and $k=k 0$ and are computed by fitting the experimental mean gain and excess noise factor for each device (with specified width) to the conventional expressions for the mean gain and excess noise factor [7]

(12) top, (13) bottom.

$$
\begin{gathered}
\langle G\rangle=\frac{1-k_{0}}{\exp \left[-1\left(1-k_{0}\right) \alpha_{0} W\right]-k_{0}} \\
F=\quad k_{0}\langle G\rangle+\left(1-k_{0}\right)\left(2-\langle G\rangle^{-} 1\right) .
\end{gathered}
$$

We seek the unique pair $(\alpha, k)$ that yields a specified experimental pair $(\langle G\rangle, F)$. This is possible by virtue of the one-to-one correspondence between the pairs $(\langle G\rangle, F)$ and $(\alpha, k)$ in the DSMT, as discussed in [4]. To illustrate the procedure, consider the sample experimental data pair $(\langle G\rangle 0, F 0)$ marked by the symbol $\times$ in Fig. 1 . We substitute the initial values of $\alpha=\alpha 0$ and $k=k 0$ in (3), (4), (9), and (10) and numerically solve these equations. This allows a trial value for the DSMT mean gain and excess noise factor to be calculated using (7) and (8) In this illustration 
the resulting pair $(\langle G\rangle, F)$, shown as encircled point 1 in Fig. 1 , is seen to underestimate the measured values. We would then increase $\alpha$ until the calculated mean gain matches $\langle G\rangle 0$ (shown as encircled point 2 in Fig. 1). From this point forward, kand $\alpha$ are concomitantly adjusted to increase the excess noise factor, bringing it closer to $F 0$, while maintaining the mean gain at $\langle G\rangle 0$. Any increase in $k$ must be accompanied by a reduction in $\alpha$ (and vice versa) to maintain the calculated mean gain at $\langle G\rangle 0$. For example, in Fig. 1, encircled point 2 lies below $(\langle G\rangle 0, F 0)$ so that $k$ must be increased which, in turn, causes $F$ to increase, while $\alpha$ is adjusted downward to a lower value to maintain the mean gain at $\langle G\rangle 0$. The results is encircled point 3 in Fig. 1 , where $F$ is now slightly overestimated. These adjustments are repeated with progressively finer changes in $\alpha$ and $k$ until $(\langle G\rangle, F) \approx(\langle G\rangle 0, F 0)$. (The relative tolerance used in our calculation for establishing convergence is 0.01 .) This completes the procedure involved in Step 2.

TABLE I Parameters of the Width-Independent (Universal) Exponential-Ionization-Coefficient Models for InP, In0.52A10.48 As, GaAs, and Al0.2 Ga0.8 As APDs

\begin{tabular}{ccccccc}
\hline \hline & & Units & $\mathrm{InP}$ & $\mathrm{In}_{0.52} \mathrm{Al}_{0.48} \mathrm{As}$ & $\mathrm{GaAs}$ & $\mathrm{Al}_{0.2} \mathrm{Ga}_{0.8} \mathrm{As}$ \\
\hline$\alpha$ & $A$ & $\mathrm{~cm}^{-1}$ & $3.01 \times 10^{6}$ & $4.17 \times 10^{6}$ & $6.01 \times 10^{6}$ & $5.39 \times 10^{6}$ \\
& $\mathcal{E}_{c}$ & $\mathrm{~V} / \mathrm{cm}$ & $2.45 \times 10^{6}$ & $2.09 \times 10^{6}$ & $2.39 \times 10^{6}$ & $2.71 \times 10^{6}$ \\
& $m$ & & 1.08 & 1.20 & 0.90 & 0.94 \\
\hline$\beta$ & $A$ & $\mathrm{~cm}^{-1}$ & $4.29 \times 10^{6}$ & $2.65 \times 10^{6}$ & $3.59 \times 10^{6}$ & $1.28 \times 10^{6}$ \\
& $\mathcal{E}_{c}$ & $\mathrm{~V} / \mathrm{cm}$ & $2.08 \times 10^{6}$ & $2.79 \times 10^{6}$ & $2.26 \times 10^{6}$ & $2.06 \times 10^{6}$ \\
& & 1.12 & 1.07 & 0.92 & 0.95 \\
\hline \hline
\end{tabular}

\begin{tabular}{|l|l|l|l|l|l|l|}
\hline & & Units & $\operatorname{InP}$ & $\operatorname{In}_{0.52} \mathrm{Al}_{0.38} \mathrm{As}$ & $\mathrm{G}_{\mathrm{a}} \mathrm{As}$ & $\mathrm{Al}_{0.2} \mathrm{Ga}_{0.8} \mathrm{As}$ \\
\hline$a$ & $A$ & $\mathrm{~cm}^{-1}$ & $3.01 \times 10^{6}$ & $4.17 \times 10^{6}$ & $6.01 \times 10^{6}$ & $5.39 \times 10^{6}$ \\
\hline & $\varepsilon_{c}$ & $\mathrm{~V} / \mathrm{cm}$ & $2.45 \times 10^{6}$ & $2.09 \times 10^{6}$ & $2.39 \times 10^{6}$ & $2.71 \times 10^{6}$ \\
\hline & $m$ & & 1.08 & 1.20 & 0.90 & 0.94 \\
\hline & $\mathrm{A}$ & $\mathrm{cm}^{-1}$ & $4.29 \times 10^{6}$ & $2.65 \times 10^{6}$ & $3.59 \times 10^{6}$ & $1.28 \times 10^{6}$ \\
\hline & $\mathcal{E}_{c}$ & $\mathrm{~V} / \mathrm{cm}$ & $2.08 \times 10^{6}$ & $2.79 \times 10^{6}$ & $2.26 \times 10^{6}$ & $2.06 \times 10^{6}$ \\
\hline & $m$ & & 1.12 & 1.07 & 0.92 & 0.95 \\
\hline
\end{tabular}

TABLE II Relative Width of Dead Space for Four Thin InP APDs of Different Multiplication-Region Widths, Producing Comparable Mean Gain. The Lower and Upper Limits of the Electric-Field Strength Produce the Lower and Upper Limits of the Mean Gain and the Relative Dead Space 


\begin{tabular}{ccccc}
\hline \hline $\begin{array}{c}\text { InP } \\
\text { Multiplication Width }(\mathrm{nm})\end{array}$ & $\begin{array}{c}\mathcal{E} \text { Field } \\
\left(\times 10^{5} \mathrm{~V} / \mathrm{cm}\right)\end{array}$ & $\begin{array}{c}\text { Mean } \\
\text { Gain }\end{array}$ & $\begin{array}{c}d_{e} / W \\
(\%)\end{array}$ & $\begin{array}{c}d_{h} / W \\
(\%)\end{array}$ \\
\hline 281 & $6.7-7.3$ & $4-20$ & $14-15$ & $15-16$ \\
317 & $5.6-6.5$ & $4-18$ & $13-15$ & $14-15$ \\
582 & $5.2-5.7$ & $4-14$ & $7.9-8.2$ & $8.8-9.1$ \\
1110 & $5.0-5.3$ & $3-14$ & $4.4-4.6$ & $4.9-5.0$ \\
\hline \hline
\end{tabular}

\begin{tabular}{|c|c|c|c|c|}
\hline $\ln P$ & $\mathcal{E}$ Field & Mean & $\mathrm{d}_{\mathrm{e}} / \mathrm{W}$ & $\mathrm{d}_{\mathrm{h}} / \mathrm{W}$ \\
\hline Multiplication Width $(\mathrm{nm})$ & $\left(\times 10^{5} \mathrm{~V} / \mathrm{cm}\right)$ & Gain & $(\%)$ & $(\%)$ \\
\hline 281 & $6.7-7.3$ & $4-20$ & $14-15$ & $15-16$ \\
\hline 317 & $5.6-6.5$ & $4-18$ & $13-15$ & $14-15$ \\
\hline 582 & $5.2-5.7$ & $4-14$ & $7.9-8.2$ & $8.8-9.1$ \\
\hline 1110 & $5.0-5.3$ & $3-14$ & $4.4-4.6$ & $4.9-5.0$ \\
\hline
\end{tabular}

\section{SECTION IV.}

\section{Results}

For each of the four materials (InP, In0.52Al0.48As, GaAs, and Al0.2Ga0.8As) we were able to find a single set of parameters $(A, E c$, and $m$ ) that satisfied the exponential model provided in (11)independent of the multiplication-region width. This enabled us to estimate the electron and hole ionization coefficients, $\alpha(E)$ and $B(E)$, respectively. Since the impact-ionization rate for holes in $\ln P$ is greater than that for electrons, the carriers were reversed in the recurrence equations, as discussed earlier. The optimized sets of width-independent parameters $A, E c$ and $m$ that yielded the best fit in the universal exponential model, for both electrons $(\alpha)$ and holes $(B)$ are provided in Table I. The improved fitting procedure used in this paper causes both the GaAs and Al0.2Ga0.8As values to differ slightly from those presented in Tables I and II of [4].

The values of $m$ are quite close to unity. Theoretically, they are known to depend on the approximation used for the electron distribution function [41]. In fact, $m$ is predicted to be exactly unity if the mean-free-path for ionizing collisions is much smaller than that for phonon collisions. This approximation is clearly most suitable at larger values of the electric field. For low values of the electric field, on the other hand, only a few "lucky" electrons are expected to attain the threshold energy necessary for impact-ionization in a single free path, and $m$ turns out to be 2 . Since the electric-field values for thin APDs are typically large, it is plausible to conclude that the observation of $m$ near unity accords with the notion that the mean-free-path for ionizing collisions is much smaller than that for phonon collisions, and this suggests that the latter can be neglected. To confirm the validity of the values of $m$ deduced from our fitting procedure, we carried out a series of model-fitting calculations in which the parameter $m$ was forced to unity. The results revealed that the $m=1$ curves were inferior to those in which $m$ was used as a fitting parameter. Indeed $m$ turns out to be the most sensitive parameter of the three in the exponential model. 


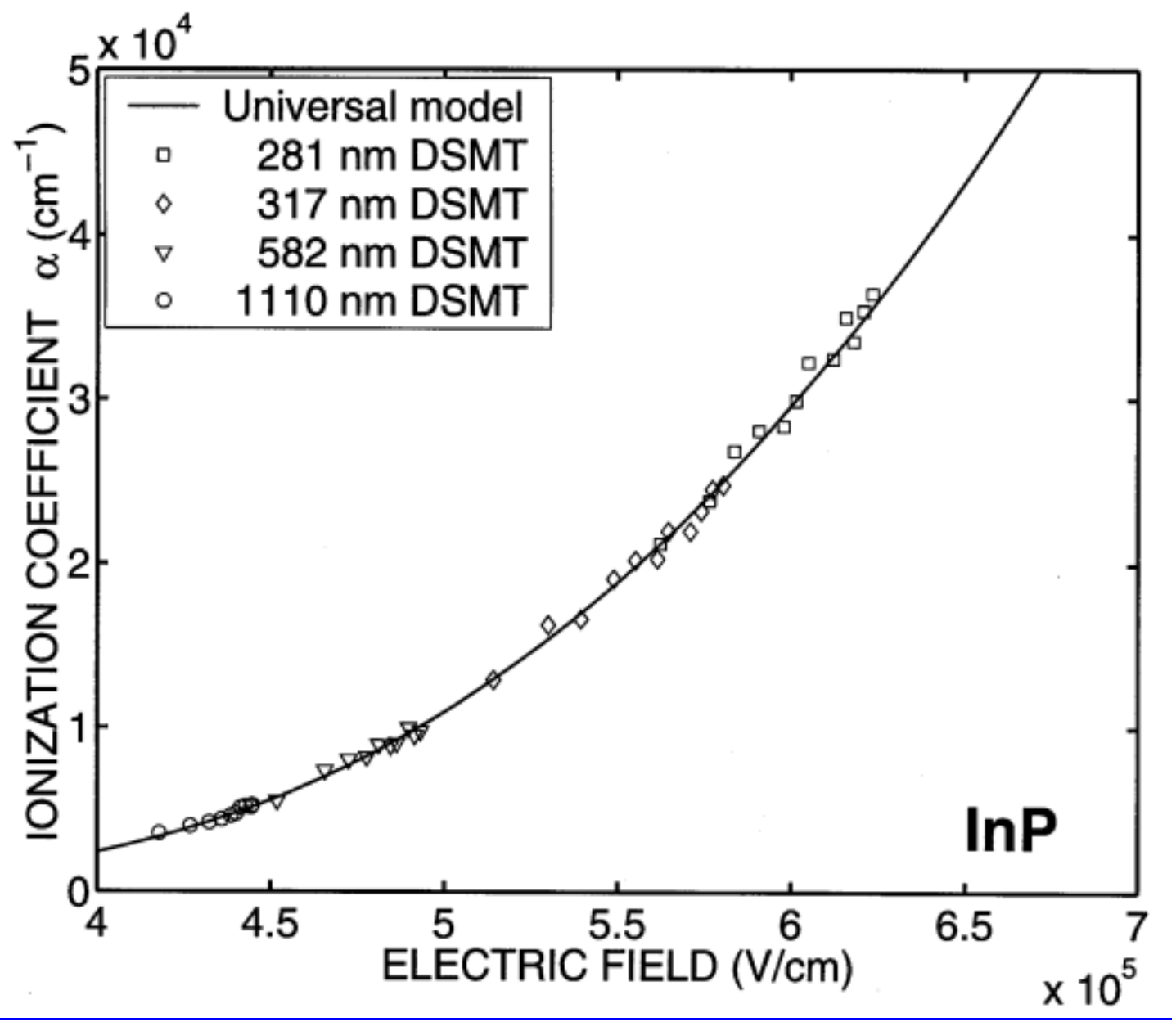

Fig. 2. Electron ionization coefficient $(\alpha)$ of InP as a function of the electric field. Symbols represent calculated values of the ionization coefficients of enabled carriers for four thin InP APDs (thickness indicated in inset), using the DSMT. The solid curve represents the universal exponential model. 


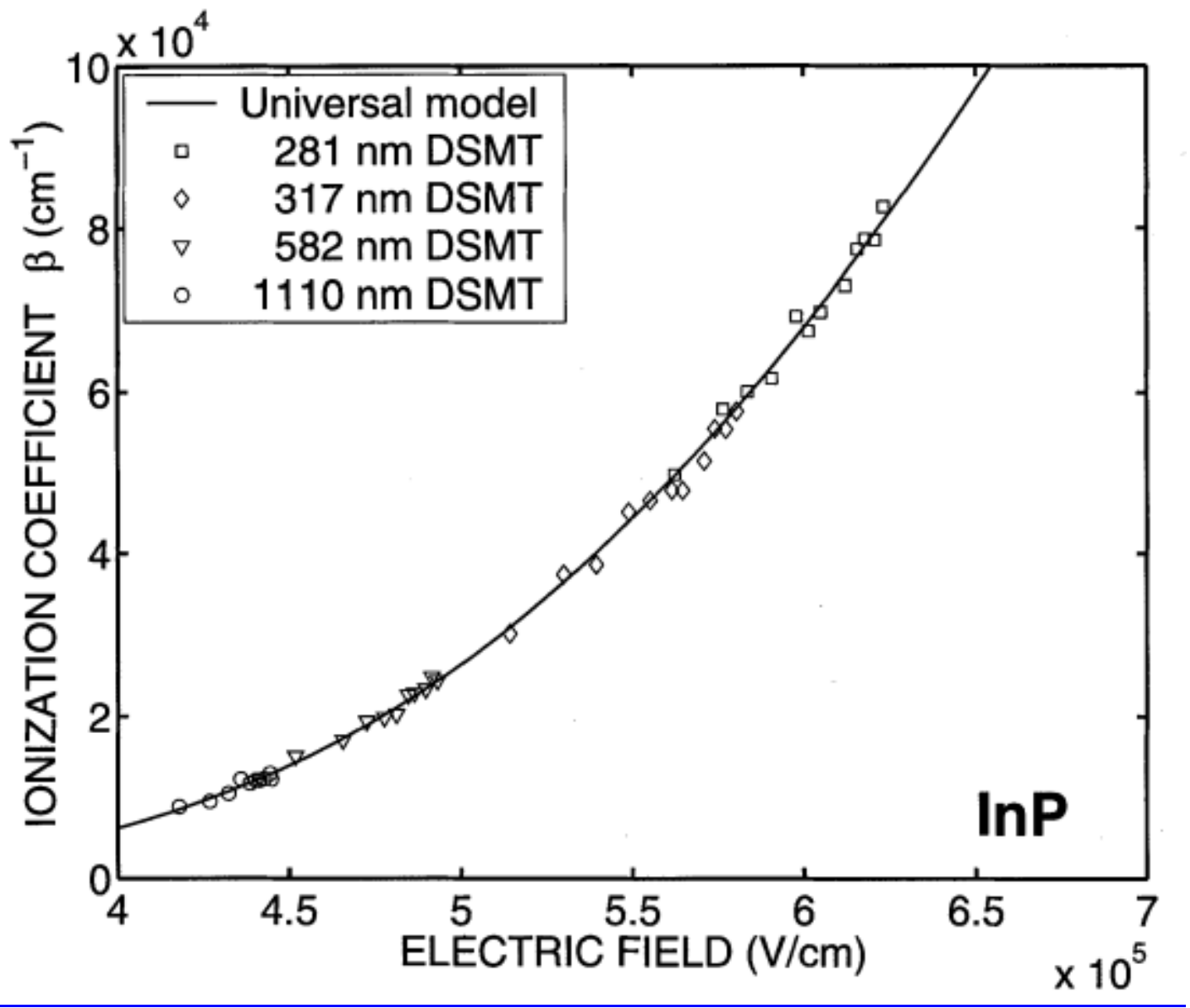

Fig. 3. Hole ionization coefficient ( 6 ) of $\operatorname{InP}$ as a function of the electric field. Plot symbols are the same as in Fig. 2. 


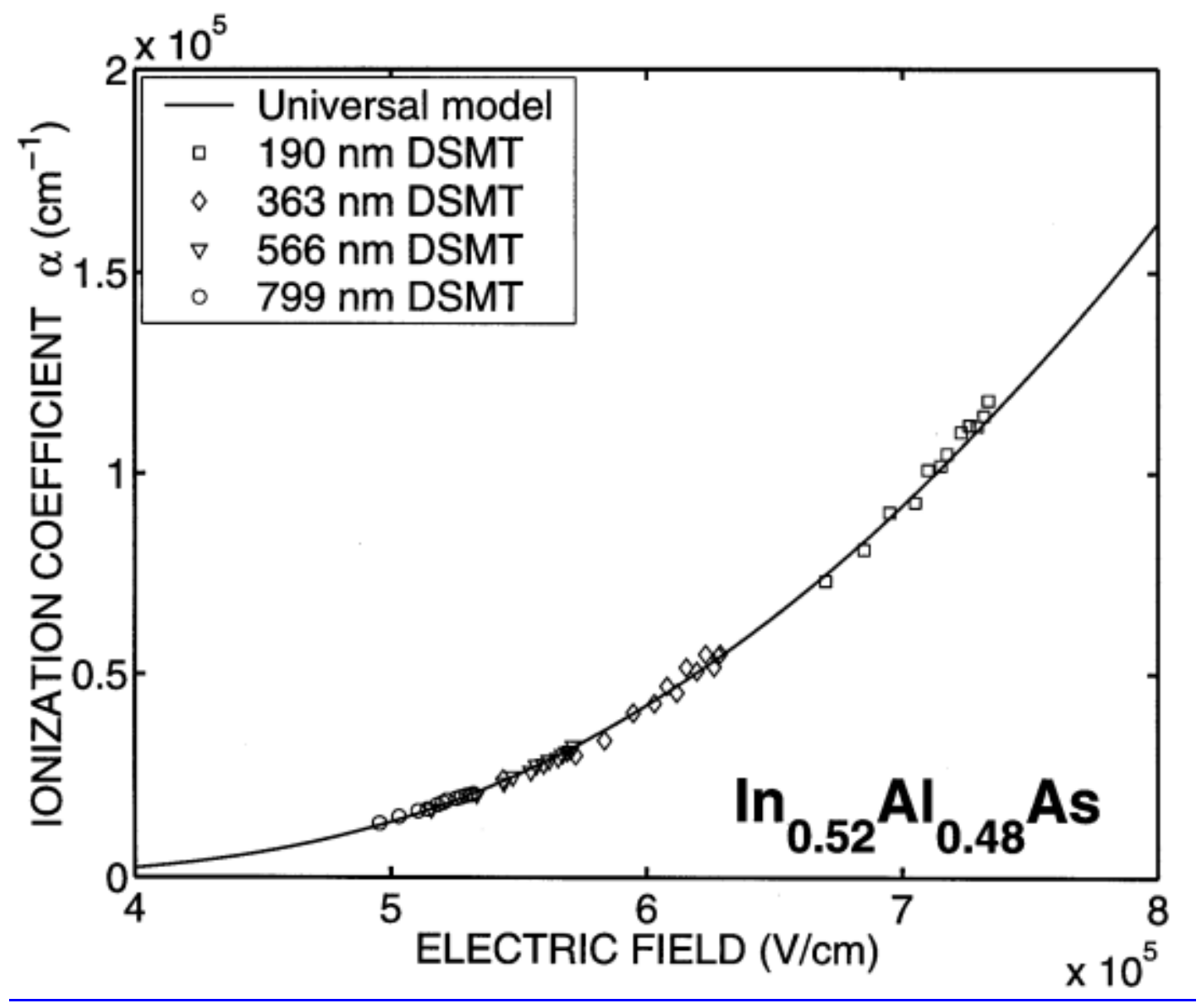

Fig. 4. Electron ionization coefficient $(\alpha)$ of In0.52Al0.48As as a function of the electric field. Symbols represent calculated values of the ionization coefficients of capable carriers for four thin In0.52Al0.48As APDs (thickness indicated in inset), using the DSMT. The solid curve represents the universal exponential model.

The electron and hole ionization coefficients for InP are displayed in Figs. 2 and 3, respectively, whereas those for In0.52Al0.48As are presented in Figs. 4 and 5, respectively. It is evident from Figs. $2-5$ that for each material, the calculated electron and hole ionization coefficients of enabled carriers $\alpha$ and 6 , respectively, (symbols), are in excellent agreement with the single exponential model, for all multiplication-region widths. This is in accord with expectation since physical principles dictate that the ionization coefficients of an enabled carrier should be material specific but independent of multiplication-region width. Similar results were obtained for the GaAs and Al0.2Ga0.8As APDs; the DSMT fitting procedure enhancement set forth in this paper provides significant improvement over the results previously reported in [4]. As in our earlier work [4], we observe that the DSMTderived ionization coefficients for GaAs, AIGaAs, and InP are higher than those derived for bulk material [30], [42], [43] (the conventional theory was used in deriving the ionization coefficient from the bulk experimental data). The authors are not aware of reported ionization coefficients for bulk InAlAs. 


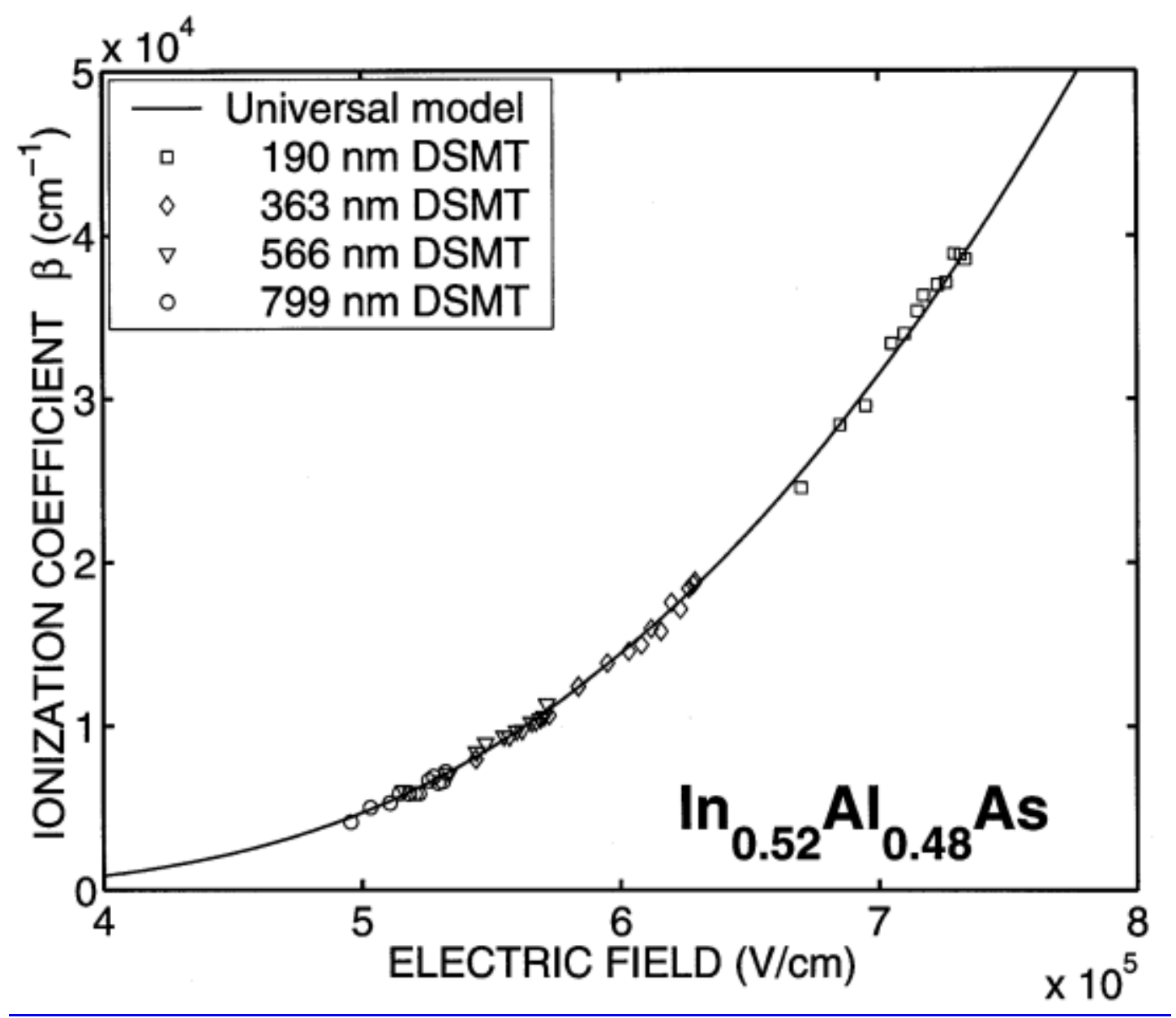

Fig. 5. Hole ionization coefficient $(B)$ of In0.52Al0.48As as a function of the electric field. Plot symbols are the same as in Fig. 4.

The relative dead space extracted from the DSMT model, which is defined as the ratio of the dead space to the multiplication-region width, is presented in Tables II and III, for InP and In0.52AI0.48As multiplication regions of different widths, respectively. Since a thin device requires a higher electric field than a thick device to provide the same mean gain, the dead space associated with a thin device is smaller than that for a thick device. Nevertheless, as is evident in Tables II and III, the relative dead space increases as the multiplication-region width is reduced. This behavior is in accord with the results reported by Li et al. [20] and Ong et al. [21], and underlies the salutary reduction in excess noise factor as multiplication-region thickness is reduced [15]. Values for the relative dead space for GaAs and AI0.2Ga0.8As APDs are close to those reported in [4].

TABLE III Relative Width of Dead Space for Four Thin In0.52 A10.48 As APDs of Different Multiplication-Region Widths, Producing Comparable Mean Gain. the Lower and Upper Limits of the Electric-Field Strength Produce the Lower and Upper Limits of the Mean Gain and the Relative Dead Space 


\begin{tabular}{ccccc}
\hline \hline $\begin{array}{c}\mathrm{In}_{0.52} \mathrm{Al}_{0.48} \mathrm{As} \\
\text { Multiplication Width }(\mathrm{nm})\end{array}$ & $\begin{array}{c}\mathcal{E} \text { Field } \\
\left(\times 10^{5} \mathrm{~V} / \mathrm{cm}\right)\end{array}$ & $\begin{array}{c}\text { Mean } \\
\text { Gain }\end{array}$ & $\begin{array}{c}d_{e} / W \\
(\%)\end{array}$ & $\begin{array}{c}d_{h} / W \\
(\%)\end{array}$ \\
\hline 190 & $5.6-6.2$ & $4-20$ & $15-16$ & $18-19$ \\
363 & $5.2-5.8$ & $3-20$ & $11-12$ & $13-14$ \\
566 & $4.5-4.9$ & $3-20$ & $6.6-7.0$ & $8.3-8.6$ \\
799 & $3.9-4.4$ & $4-20$ & $5.4-5.7$ & $6.3-6.6$ \\
\hline \hline
\end{tabular}

\begin{tabular}{|c|c|c|c|c|}
\hline In0.52AI0.48As & $\mathcal{E}$ Field & Mean & $\mathrm{d}_{\mathrm{e}} / \mathrm{W}$ & $\mathrm{d}_{\mathrm{h}} / \mathrm{W}$ \\
\hline Multiplication Width $(\mathrm{nm})$ & $\left(\times 10^{5} \mathrm{~V} / \mathrm{cm}\right)$ & Gain & $(\%)$ & $(\%)$ \\
\hline 190 & $5.6-6.2$ & $4-20$ & $15-16$ & $18-19$ \\
\hline 363 & $5.2-5.8$ & $3-20$ & $11-12$ & $13-14$ \\
\hline 566 & $4.5-4.9$ & $3-20$ & $6.6-7.0$ & $8.3-8.6$ \\
\hline 799 & $3.9-4.4$ & $4-20$ & $5.4-5.7$ & $6.3-6.6$ \\
\hline
\end{tabular}

TABLE IV Electron and Hole Ionization Threshold Energies of InP, In0.52 Al0.48 As, GaAs, and Al0.2 Ga0.8 As. Initial Values were Obtained From the Sources Cited. DSMT Values are Determined from the Best Fit to GainNoise Data for Thin APDs

\begin{tabular}{ccccccccc}
\hline \hline & InP & InP & InAlAs & InAlAs & GaAs & GaAs & AlGaAs & AlGaAs \\
& Initial [44] & DSMT & Initial [45] & DSMT & Initial [34] & DSMT & Initial [31] & DSMT \\
\hline$E_{\text {ie }}$ & $1.84 \mathrm{eV}$ & $2.05 \mathrm{eV}$ & $1.90 \mathrm{eV}$ & $2.15 \mathrm{eV}$ & $1.70 \mathrm{eV}$ & $1.90 \mathrm{eV}$ & $1.84 \mathrm{eV}$ & $2.04 \mathrm{eV}$ \\
$E_{\text {ih }}$ & $1.64 \mathrm{eV}$ & $2.20 \mathrm{eV}$ & $2.00 \mathrm{eV}$ & $2.30 \mathrm{eV}$ & $1.40 \mathrm{eV}$ & $1.55 \mathrm{eV}$ & $1.94 \mathrm{eV}$ & $2.15 \mathrm{eV}$ \\
\hline \hline
\end{tabular}

\begin{tabular}{|l|l|l|l|l|l|l|l|l|}
\hline & InP & InP & InAIAs & InAIAs & GaAs & GaAs & AIGaAs & AIGaAs \\
\hline & Initial [44] & DSMT & Initial [45] & DSMT & Initial [34] & DSMT & Initial [31] & DSMT \\
\hline $\mathrm{E}_{i e}$ & $1.84 \mathrm{eV}$ & $2.05 \mathrm{eV}$ & $1.90 \mathrm{eV}$ & $2.15 \mathrm{eV}$ & $1.70 \mathrm{eV}$ & $1.90 \mathrm{eV}$ & $1.84 \mathrm{eV}$ & $2.04 \mathrm{eV}$ \\
\hline $\mathrm{E}_{i h}$ & $1.64 \mathrm{eV}$ & $2.20 \mathrm{eV}$ & $2.00 \mathrm{eV}$ & $2.30 \mathrm{eV}$ & $1.40 \mathrm{eV}$ & $1.55 \mathrm{eV}$ & $1.94 \mathrm{eV}$ & $2.15 \mathrm{eV}$ \\
\hline
\end{tabular}




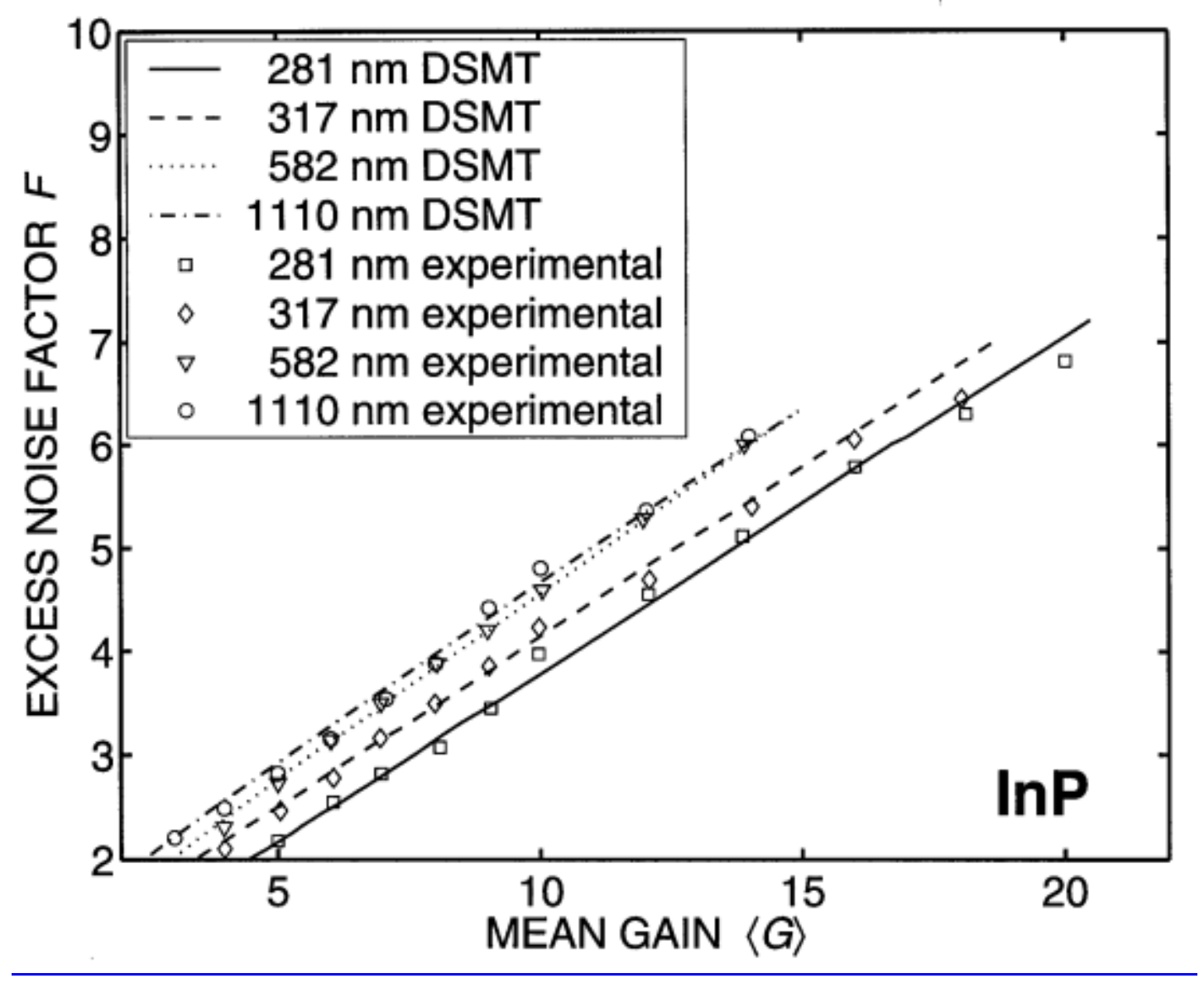

Fig. 6. Comparison of the DSMT-predicted and the experimental excess noise factor Fversus mean gain $\langle G\rangle$ for four thin InP APDs with different multiplication-region widths. Symbols represent experimental data and curves represent predictions using the DSMT.

The established values for the electron and hole ionization energies, Eie and Eih, and the values returned for the best fit of the DSMT to the gain-noise data, are provided in Table IV. It is clear that the effective ionization threshold energies estimated via the DSMT are larger than the theoretically established values [31], [34], [44], [45] for both electrons and holes in all four materials. Based on these results one could speculate that the estimates returned by the DSMT include phononscattering energy loss, but this conclusion would be unfounded. 


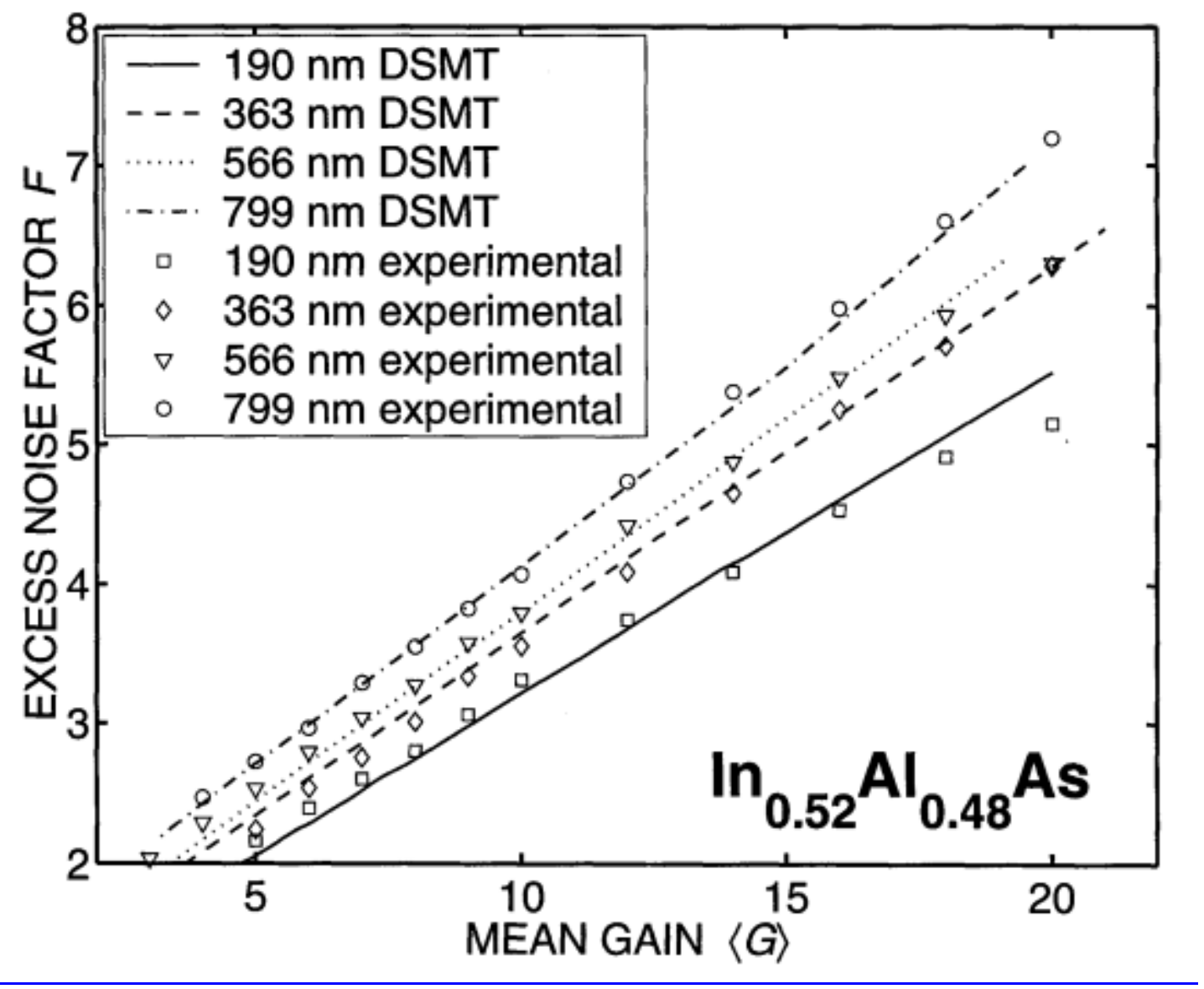

Fig. 7. Comparison of the DSMT-predicted and the experimental excess noise factor Fversus mean gain $\langle G\rangle$ for four thin In0.52AI0.48As APDs with different multiplication-region widths. Symbols represent experimental data and curves represent predictions using the DSMT. 


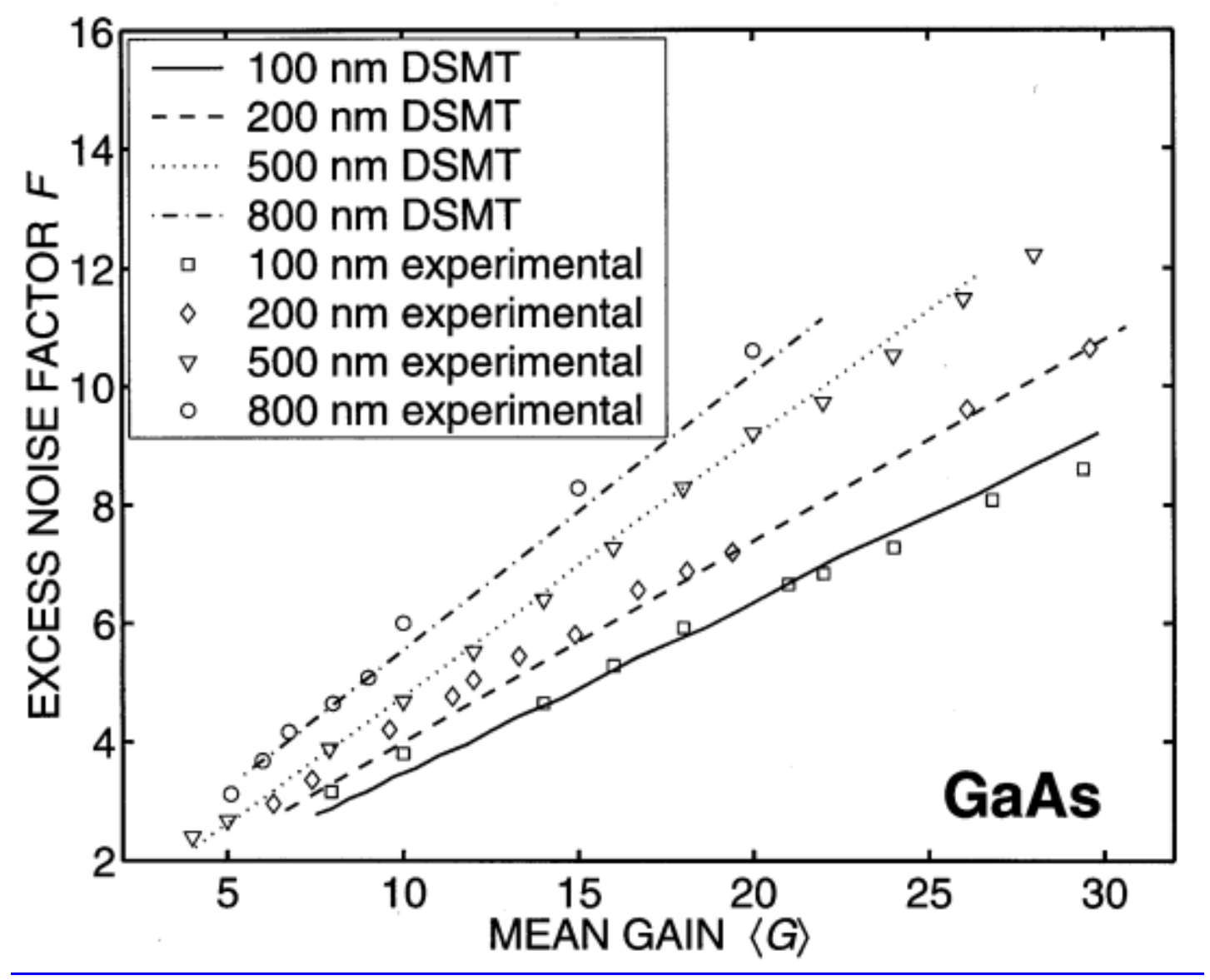

Fig. 8. Comparison of the DSMT-predicted and the experimental excess noise factor Fversus mean gain $\langle G\rangle$ for four thin GaAs APDs with different multiplication-region widths. Symbols represent experimental data and curves represent predictions using the DSMT. Though the data are identical to those presented in [4], an improved fitting procedure has been used.

The initial values used in our calculations are quite removed from those that emerge from the most complete theory that is currently available [33]. According to Bude and Hess [33], who are likely to have the most accurate results, qualitative estimates of the electron ionization threshold for GaAs and InP, with respect to the bandgap energy, are expected to be in the vicinity of $1.9 \mathrm{eV}$ and $2.3 \mathrm{eV}$, respectively. On the other hand, the DSMT electron ionization threshold values derived from experimental data turn out to be $1.9 \mathrm{eV}$ and $2.1 \mathrm{eV}$, respectively, for these two materials [see Table II]. Thus the electron ionization threshold obtained by Bude and Hess [33] is identical for GaAs and within $10 \%$ for InP compared to that derived by DSMT. (Clearly, fitting the DSMT to the experimental data would have been facilitated had we started with their value). Attempts to force the InP electron ionization threshold energy to the Bude and Hess value of $2.3 \mathrm{eV}$ failed to produce as good a fit to the experimental data as the $2.1 \mathrm{eV}$ result reported in Table IV. However, it is not surprising to find that the effective ionization threshold values are not identical to the theoretical values. The small deviation accounts for errors resulting from the approximations involved in the simple hard-threshold dead-space model. The proximity of these two theoretical and DSMT derived ionization threshold values nonetheless justifies our use of (1) and (2) and suggests that the phonon scattering loss may indeed be ignored. 


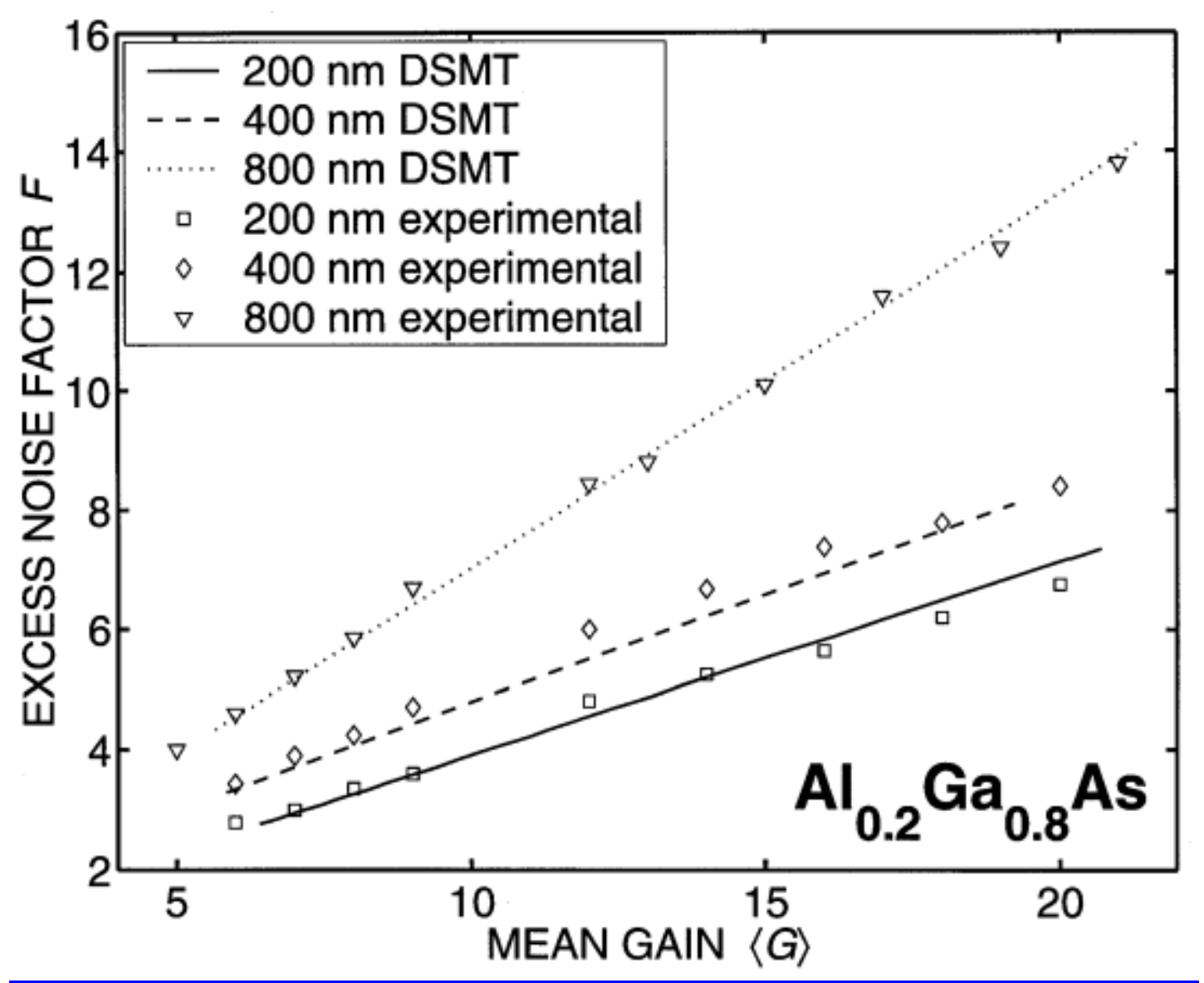

Fig. 9. Comparison of the DSMT-predicted and the experimental excess noise factor Fversus mean gain $\langle G\rangle$ for three thin AI0.2Ga0.8As APDs with different multiplication-region widths. Symbols represent experimental data and curves represent predictions using the DSMT. Though the data are identical to those presented in [4], an improved fitting procedure has been used.

Since Bude and Hess [33] did not provide ionization threshold energies for electrons in AlGaAs and InAlAs, nor did they provide ionization threshold energies for holes in any material, it was not possible to compare our results with theirs for all entries in Table IV. In any case, the effective ionization threshold obtained from the DSMT should be independent of the initial value used for the computations.

Finally, the excess noise factor versus mean gain is presented in Figs. $6-9$ for InP, In0.52Al0.48As, GaAs, and Al0.2Ga0.8As APDs, respectively. Symbols indicate experimental measurements while curves correspond to predictions of the DSMT. The agreement is excellent in all cases. It is apparent that optimization over the ionization-threshold energy provides improved agreement of theory with experiment as is evident by comparison of Figs. 8 and 9, for GaAs and Al0.2Ga0.8As, respectively, with [4]. The fits in [4] made use of threshold energies taken directly from the literature [31], [34].

\section{SECTION V.}

\section{Conclusion}

We have provided a technique for significantly improving the usefulness of the DSMT [4], [15] for analyzing avalanche-photodiode data and for determining material-specific ionization coefficients of (enabled) carriers 
that have traveled a distance greater than the dead space. The ionization coefficients are calculated directly from excess-noise-factor measurements, without having to resort to simulation of any kind. This improvement is achieved by adjusting the theoretical estimates of the threshold energy reported in the literature to generate effective ionization threshold energies. The use of an effective threshold energy compensates for the inaccuracies in the theoretical threshold-energy estimates and the limitations imposed by the simple hardthreshold dead-space model. The estimated effective threshold energies are generally found to be higher than the theoretical values reported in the literature. It is shown that the DSMT provides an excellent description of excess noise factor versus mean-gain data for all four materials examined, for all multiplication-region widths.

Finally, we point out that the frequency response and breakdown-voltage characteristics of thin APDs can be readily and accurately determined [46]-[47] [48] by making use of the ionization coefficients obtained by the methods reported here.

\section{References}

1. B. E. A. Saleh, M. C. Teich, "17" in Fundamentals of Photonics, New York:Wiley, 1991.

2. M. C. Teich, K. Matsuo, B. E. A. Saleh, "Excess noise factors for conventional and superlattice avalanche photodiodes and photomultiplier tubes", IEEE J. Quantum Electron., vol. QE-22, pp. 1184-1193, 1986.

3. P. Yuan, C. C. Hansing, K. A. Anselm, C. V. Lenox, H. Nie, A. L. Holmes Jr., B. G. Streetman, J. C. Campbell, "impact-ionization characteristics of IIIV semiconductors for a wide range of multiplication region thicknesses", IEEE J. Quantum Electron., vol. 36, pp. 198-204, 2000.

4. M. A. Saleh, M. M. Hayat, B. E. A. Saleh, M. C. Teich, "Dead-space-based theory correctly predicts excess noise factor for thin GaAs and AIGaAs avalanche photodiodes", IEEE Trans. Electron Devices, vol. 47, pp. 625-633, Mar. 2000.

5. G. S. Kinsey, C. C. Hansing, A. L. Holmes Jr., B. G. Streetman, J. C. Campbell, A. G. Dentai, " Waveguide $\backslash \$$ hbox $\{\mathrm{In}\} \_\{0.53\}$ hbox $\{\mathrm{Ga}\} \_\{0.47\} \backslash \$$ As- $\backslash \$$ hbox $\{\mathrm{In}\} \_\{0.52\} \backslash \$ \backslash \$$ hbox $\{A \mid\} \_\{0.48\} \backslash \$$ As avalanche photodiode ", IEEE Photon. Technol. Lett., vol. 12, pp. 416-418, 2000.

6. C. Lenox, H. Nie, P. Yuan, G. Kinsey, A. L. Homles Jr., B. G. Streetman, J. C. Campbell, "Resonant-cavity InGaAsInAIAs avalanche photodiodes with gain-bandwidth product of $290 \mathrm{GHz}$ ", IEEE Photon. Technol. Lett., vol. 11, pp. 1162-1164, 1999.

7. R. J. McIntyre, "Multiplication noise in uniform avalanche photodiodes", IEEE Trans. Electron Devices, vol. ED13, pp. 164-168, Jan. 1966.

8. R. Chin, N. Holonyak, G. E. Stillman, J. Y. Tang, K. Hess, "Impact ionization in multilayered heterojunction structures", Electron. Lett., vol. 16, pp. 467-469, 1980.

9. G. F. Williams, F. Capasso, W. T. Tsang, "The graded bandgap multilayer avalanche photodiode: A new lownoise detector", IEEE Electron Device Lett., vol. EDL-3, pp. 71-73, Mar. 1982.

10. N. Z. Hakim, B. E. A. Saleh, M. C. Teich, "Generalized excess noise factor for avalanche photodiodes of arbitrary structure", IEEE Trans. Electron Devices, vol. 37, pp. 599-610, Mar. 1990.

11. K. Matsuo, M. C. Teich, B. E. A. Saleh, "Noise properties and time response of the staircase avalanche photodiode", IEEE Trans. Electron Devices, vol. ED-32, pp. 2615-2623, Dec. 1985. 
12. Y. Okuto, C. R. Crowell, "Ionization coefficients in semiconductors: A nonlocalized property", Phys. Rev. B Condens. Matter, vol. 10, pp. 4284-4296, 1974.

13. R. A. La Violette, M. C. Stapelbroek, "A non-Markovian model of avalanche gain statistics for solid-state photomultiplier", J. Appl. Phys., vol. 65, pp. 830-836, 1989.

14. B. E. A. Saleh, M. M. Hayat, M. C. Teich, "Effect of dead space on the excess noise factor and time response of avalanche photodiodes", IEEE Trans. Electron Devices, vol. 37, pp. 1976-1984, Oct. 1990.

15. M. M. Hayat, B. E. A. Saleh, M. C. Teich, "Effect of dead space on gain and noise of double-carriermultiplication avalanche photodiodes", IEEE Trans. Electron Devices, vol. 39, pp. 546-552, Mar. 1992.

16. M. M. Hayat, W. L. Sargeant, B. E. A. Saleh, "Effect of dead space on gain and noise in Si and GaAs avalanche photodiodes", IEEE J. Quantum Electron., vol. 28, pp. 1360-1365, 1992.

17. M. M. Hayat, Z. Chen, M. A. Karim, "An analytical approximation for the excess noise factor of avalanche photodiodes with dead space", IEEE Electron Device Lett., vol. 20, pp. 344-347, Oct. 1999.

18. A. Spinelli, A. L. Lacaita, "Mean gain of avalanche photodiodes in a dead space model", IEEE Trans. Electron Devices, vol. 43, pp. 23-30, Jan. 1996.

19. R. J. Mclntyre, "A new look at impact-ionizationPart I: A theory of gain noise breakdown probability and frequency response", IEEE Trans. Electron Devices, vol. 46, pp. 1623-1631, Aug. 1999.

20. K. F. Li, D. S. Ong, J. P. R. David, G. J. Rees, R. C. Tozer, P. N. Robson, R. Grey, " Avalanche multiplication noise characteristics in thin GaAs $\backslash \$ p^{\wedge}\{+\}$ hbox $\{--\}$ ihbox $\{--\} n^{\wedge}+\backslash \$$ diodes ", IEEE Trans. Electron Devices, vol. 45, pp. 2102-2107, Nov. 1998.

21. D. S. Ong, K. F. Li, G. J. Rees, G. M. Dunn, J. P. R. David, P. N. Robson, " A Monte Carlo investigation of multiplication noise in thin $\backslash \$ p^{\wedge}\{+\}$ hbox $\{--\}$ ihbox $\{--\} n^{\wedge}+\backslash \$ G a A s$ avalanche photodiodes ", IEEE Trans. Electron Devices, vol. 45, pp. 1804-1810, Oct. 1998.

22. P. Yuan, K. A. Anselm, C. Hu, H. Nie, C. Lenox, A. L. Holmes, B. G. Streetman, J. C. Campbell, R. J. Mclntyre, "A new look at impact-ionization-Part II: Gain and noise in short avalanche photodiodes", IEEE Trans. Electron Devices, vol. 46, pp. 1632-1639, Aug. 1999.

23. S. A. Plimmer, J. P. R. David, R. Grey, G. J. Rees, " Avalanche multiplication in $\backslash \$$ hbox $\{A \mid\} \_\{x\} h b o x\{G a\} \_\{1-x\} \backslash \$$ As ( \\$x=0\\$ to 0.60) ", IEEE Trans. Electron Devices, vol. 47, pp. 1089-1097, May 2000.

24. J. N. Hollenhorst, "A theory of multiplication noise", IEEE Trans. Electron Devices, vol. 37, pp. 781-788, Apr. 1990.

25. J. C. Campbell, S. Chandrasekhar, W. T. Tsang, G. J. Qua, B. C. Johnson, "Multiplication noise of widebandwidth InP/InGaAsP/InGaAs avalanche photodiodes", J. Lightwave Technol., vol. 7, pp. 473-477, 1989.

26. V. Chandramouli, C. M. Maziar, J. C. Campbell, "Design considerations for high performance avalanche photodiode multiplication layers", IEEE Trans. Electron Devices, vol. 41, pp. 648-654, Mar. 1994.

27. C. Hu, K. A. Anselm, B. G. Streetman, J. C. Campbell, "Noise characteristics of thin multiplication region GaAs avalanche photodiodes", Appl. Phys. Lett., vol. 69, pp. 3734-3736, 1996.

28. K. A. Anselm, P. Yuan, C. Hu, C. Lenox, H. Nie, G. Kinsey, J. C. Campbell, B. G. Streetman, "Characteristics of GaAs and AlGaAs homojunction avalanche photodiodes with thin multiplication regions", Appl. Phys. Lett., vol. 71, pp. 3883-3885, 1997. 
29. C. Lenox, P. Yuan, H. Nie, O. Baklenov, C. Hansing, J. C. Campbell, A. L. Holmes Jr., B. G. Streetman, "Thin multiplication region InAlAs homojunction avalanche photodiodes", Appl. Phys. Lett., vol. 73, pp. 783-784, 1998.

30. G. E. Bulman, V. M. Robbins, K. F. Brennan, K. Hess, G. E. Stillman, "Experimental determination of impactionization coefficients in (100) GaAs", IEEE Electron Device Lett., vol. EDL-4, pp. 181-185, May 1983.

31. J. P. R. David, J. S. Marsland, H. Y. Hall, G. Hill, N. J. Mason, M. A. Pate, J. S. Roberts, P. N. Robson, J. E. Sitch, R. C. Woods, "Measured ionization coefficients in \\$hbox $\{G a\} \_\{1-x\}$ hbox $\{A l\} \_x$ hbox $\{A s\} \backslash \$$ ", Proc. IPCS 74, pp. 247-252, 1985.

32. A. Spinelli, A. Pacelli, A. L. Lacaita, "Dead space approximation for impact-ionization in silicon", Appl. Phys. Lett., vol. 69, pp. 3707-3709, 1996.

33. J. Bude, K. Hess, "Thresholds of impact-ionization in semiconductors", J. Appl. Phys., vol. 72, pp. 3554-3561, 1992.

34. C. L. Anderson, C. R. Crowell, "Threshold energies for electronhole pair production by impact-ionization in semiconductors", Phys. Rev. B Condens. Matter, vol. 5, pp. 2267-2272, 1972.

35. B. I. Cantor, M. C. Teich, "Dead-time-corrected photocounting distributions for laser radiation", J. Opt. Soc. Amer., vol. 65, pp. 786-791, 1975.

36. B. E. A. Saleh, Photoelectron Statistics, New York:Springer-Verlag, pp. 272-279, 1978.

37. P. R. Prucnal, M. C. Teich, "Refractory effects in neural counting processes with exponentially decaying rates", IEEE Trans. Syst. Man Cybern., vol. SMC-13, pp. 1028-1033, 1983.

38. M. C. Teich, L. Matin, B. I. Cantor, "Refractoriness in the maintained discharge of the cat's retinal ganglion cell", J. Opt. Soc. Amer., vol. 68, pp. 386-402, 1978.

39. M. C. Teich, P. Diament, "Relative refractoriness in visual information processing", Biol. Cybern., vol. 38, pp. 187-191, 1980.

40. J. L. Moll, N. Meyer, "Secondary multiplication in silicon", Solid State Electron., vol. 3, pp. 155-161, 1961.

41. R. K. Willardson, A. C. Beer, "Avalanche photodiodes" in Semiconductors and Semimetals, New York:Academic, vol. 12, pp. 291-393, 1977.

42. V. M. Robbins, S. C. Smith, G. E. Stillman, "Impact ionization in $\backslash \$$ hbox $\{A \mid\} \_\{x\}$ hbox $\{G a\} \_\{1-x\} \backslash \$$ As for $\backslash \$ x=0.1-0.4 \backslash \$$ ", Appl. Phys. Lett., vol. 52, pp. 296-298, 1988.

43. L. W. Cook, G. E. Bulman, G. E. Stillman, Appl. Phys. Lett., vol. 40, pp. 589-589, 1982.

44. T. P. Pearsall, "Threshold energies for impact-ionization by electrons and holes in InP", Appl. Phys. Lett., vol. 35, pp. 168-170, 1979.

45. I. Watanabe, T. Toshitaka, K. Taguchi, "Monte Carlo simulation of impact-ionization rates in InAIAsInGaAs square and graded barrier superlattices", IEEE J. Quantum Electron., vol. 31, pp. 1826-1834, 1995.

46. M. M. Hayat, B. E. A. Saleh, "Statistical properties of the impulse response function of double-carrier multiplication avalanche photodiodes including the effect of dead space", J. Lightwave Technol., vol. 10, pp. 1415-1425, 1992. 
47. M. M. Hayat, M. A. Saleh, M. C. Teich, B. E. A. Saleh, "Dead-space-theory predictions of excess-noise factor breakdown voltage and frequency response for thin avalanche photodiodes" in Photonics West 2001 Physics and Simulation of Optoelectronic Devices IX (OE09), CA, San Jose, 2001.

48. M. A. Saleh, M. M. Hayat, O. Kwon, A. L. Holmes, Jr., J. C. Campbell, B. E. A. Saleh, M. C. Teich, "Breakdown voltage in thin IIIV avalanche photodiodes", Appl. Phys. Lett. 